\title{
Quadratic Forms and Klauder's Phenomenon: A Remark on Very Singular Perturbations
}

\author{
BARRY SIMON $*,+$ \\ Départment de Physique à Luminy, Université d'Aix-Marseille II, Marseille, France \\ Communicated by G. A. Hunt
}

Reccived April 2, 1973

\begin{abstract}
We give a general analysis of a class of pairs of positive self-adjoint operators $A$ and $B$ for which $A+\lambda B$ has a limit (in strong resolvent sense) as $\lambda \downarrow 0$ which is an operator $A_{p} \neq A$ !
\end{abstract}

Recently, Klauder [4] has discussed the following example: Let $A$ be the operator $-\left(d^{2} / d x^{2}\right)+x^{2}$ on $L^{2}(\mathbb{R}, d x)$ and let $B=|x|^{-3}$. The eigenvectors and eigenvalues of $A$ are, of course, well known to be the Hermite functions, $H_{n}(x), n=0,1, \ldots$ and $\mathscr{E}_{n}=2 n+1$. Klauder then considers the eigenvectors of $A+\lambda B(\lambda>0)$ by manipulations with the ordinary differential equation (we consider the domain questions, which Klauder ignores, below). He finds that the eigenvalues $E_{n}(\lambda)$ and eigenvectors $\psi_{n}(\lambda)$ do not converge to $\mathscr{E}_{n}$ and $H_{n}$ but rather

$$
\left.\begin{array}{l}
\left.\begin{array}{l}
\left.\psi_{n}(\lambda) \rightarrow(\operatorname{sgn} x) H_{n+1}\right\} \\
E_{n}(\lambda) \rightarrow \mathscr{E}_{n+1} \\
\psi_{n}(\lambda) \rightarrow H_{n} \\
E_{n}(\lambda) \rightarrow \mathscr{E}_{n}
\end{array}\right\} \quad n=0,2, \ldots \\
\end{array}\right\}
$$

We wish to discuss in detail the general phenomena which Klauder has uncovered. We freely use the techniques of quadratic forms and strong resolvent convergence; see e.g. [3], [5].

Once one decides to analyze Klauder's phenomenon in the language of quadratic forms, the phenomenon is quite easy to understand and control. In fact, the theory is implicit in Kato's book [3, VIII.3].

* Permanent Address: Departments of Mathematics and Physics, Princeton University, Princeton, New Jersey 08540.

+ A. Sloan Foundation Fellow. 
It is, thus, with some hesitation that we write this note. However, because the phenomenon is so striking, it seems worthwhile to point out the mechanism behind it.

The first question we must face is the definition of $A+\lambda B$. We will suppose that $A$ and $B$ are positive self-adjoint operators on a separable Hilbert space $\mathscr{H}$ with form domains $Q(A)$ and $Q(B)$. If $\lambda>0$ and if $Q(A) \cap Q(B) \equiv Q$ is dense in $\mathscr{H}$, then

$$
\psi, \phi \mapsto\left(A^{\frac{1}{2}} \psi, A^{\frac{1}{2}} \phi\right)+\lambda\left(B^{\frac{1}{2}} \psi, B^{\frac{1}{2}} \phi\right)
$$

is easily secn to be a closed quadratic form on $Q$. There is, thus, a unique self-adjoint operator so that this form is the form associated to that self-adjoint operator [5, Theorem VIII.15]. Henceforth (except for Example 2 below), $A+\lambda B$ will denote this operator. Our main result tells us that Klauder's phenomena occurs if and only if $Q(A) \cap Q(B)$ is not a form core for $A$.

Theorem 1. Let $A$ and $B$ be positive self-adjoint operators with $Q(A) \cap Q(B)$ dense. Let $A_{p}$ be the (unique) positive self-adjoint operator whose associated quadratic form is the closure of the form

$$
\psi, \phi \mapsto\left(A^{\frac{1}{2}} \psi, A^{\frac{1}{2}} \phi\right)
$$

on the domain $Q(A) \cap Q(B)$. For $\lambda>0$, define $A+\lambda B$ as a sum of quadratic forms. Then as $\lambda \downarrow 0, A+\lambda B$ converges to $A_{p}$ in strong resolvent sens.

Proof. Follows from Theorem VIII.3.6 or VIII.3.11 of Kato [3]. The connection between eigenvalues of $A+\lambda B$ and those of $A_{p}$ is quite simple.

Theorem 2. Let $A, B, A_{p}$ be as in 'Theorem 1. Suppose that

$$
\Sigma=\inf \sigma_{\mathrm{ess}}\left(A_{p}\right)
$$

and that $A_{p}$ has at least $m$ eigenvalues below $\Sigma$. Let $E_{1}, \ldots, E_{m}$ be the $m$ lowest eigenvalues for $A_{p}$. Then, for all sufficiently small $\lambda, A+\lambda B$ has at least $m$ eigenvalues $E_{1}(\lambda), \ldots, E_{m}(\lambda)$ below $\Sigma(\lambda)$ and

$$
\lim _{\lambda \downarrow 0} E_{i}(\lambda)=E_{i}, \quad i=1, \ldots, m .
$$

Proof. See [3, Theorem VIII.3.15] or alternately [2] or [6, Appen$\operatorname{dix}$ C].

Let us close with a few examples. 
EXAMPLE 1.

$$
A=-\frac{d^{2}}{d x^{2}}, \quad B=|x|^{-\alpha} \text { on } L^{2}(\mathbb{R}, d x) .
$$

If $-2 \leqslant \alpha<1$, then $B$ is a small form perturbation of $A$ (see e.g. [4]) so it is easy to prove that $A+\lambda B$ converges to $A$ in norm resolvent sense as $\lambda \downarrow 0$. This last fact also holds if $\alpha<-2$ by a more complicated argument (see [6]). If $\alpha \geqslant 1$, we have a different situation. For every $\varphi \in Q(A)$ is Hölder continuous and, thus,

$$
\int|\varphi(x)|^{2}|x|^{-\alpha} d x<\infty
$$

implies that $\varphi(0)=0$. Thus, $Q(A) \cap Q(B) \subset\{\varphi \in D(A) \mid \varphi(0)=0\}$ so that $A_{p} \neq A$ but is instead $x^{2}$ plus the operator $-d^{2} / d x^{2}$ with Dirichlet boundary conditions at $x=0$. For both $A_{p}$ and $A+\lambda B$, $L^{2}((0, \infty), d x)$ is an invariant subspace.

EXAMPLE 2. One can ask a slightly different question about $A+\lambda B$ if $D(A) \cap D(B)$ is dense. Rather than ask if $A+\lambda B$ defined as a sum of forms converges to $A$ as $\lambda \downarrow 0$, one can ask if there is some family of self-adjoint extensions of $A+\lambda B \wedge D(A) \cap D(B)$ which converges to $A$ as $\lambda \downarrow 0$. In Example 1, if $\alpha>2$ there exists no such family since $A+\lambda B$ is essentially self-adjoint and its closure is the form sum (this follows from the Weyl limit point method [1] or by other methods [7]). But for $1 \leqslant \alpha \leqslant 2$, such families do exist (see [4]), and Klauder's phenomenon is not forced upon us if we look at extensions of operator sums rather than the form sum. This explains why Klauder chooses $\alpha=2$ as the borderline while Example 1 suggests $\alpha=1$ as borderline.

EXAMPLE 3.

$$
A=-\Delta+|x|^{2}, \quad B=|x|^{-\alpha}\left\{\begin{array}{l}
\text { on } L^{2}\left(\mathbb{R}^{n}, d x\right), \\
n \geqslant 2 .
\end{array}\right.
$$

It is known that $\left\{\psi \mid \psi \in C_{0}{ }^{\infty}, \psi \equiv 0\right.$ near 0$\}$ is a form core for $-\Delta$ in this case, so $A_{p}=A$. Thus, Klauder's phenomenon (in case $A$ is a harmonic oscillator) requires $B$ to have a codimension 1 singularity. As Klauder [4] remarks, this is most conviently understood in terms of Weiner path integrals (more precisely Ornstein-Uhlenbeck path integrals). 
Example 4. It is fitting to conclude with an example suggered by D. W. Robinson (private communication) which shows how unsuited the form sum can be. Let $A$ be a smooth bounded set in $\mathbb{R}^{n}$ and let $\mathscr{H}=L^{2}\left(\Lambda, d^{n} x\right)$. Let $A=-\Delta$ with Neumann boundary conditions and $B=-\Delta$ with Dirichlet boundary conditions. Then $A+\lambda B=$ $(1+\lambda) B$, so $A+\lambda B \downarrow B \neq A$ as $\lambda \downarrow 0$.

\section{ACKNOWLEDGMENTS}

It is a pleasure to thank D. W. Robinson and J. Klauder for valuable discussions and D. Kastler for the hospitality of the Départment de Physique à Luminy.

\section{REFERENCES}

1. E. A. Coddington And N. Levinson, "Theory of Ordinary Differential Equations," Mc Graw-Hill, New York Toronto London, 1945.

2. W. M. Greenlee, Singular perturbations of eigenvalues Arch. Rat. Mech. Anal. 34 (1969), 143-164.

3. T. Kato, "Perturabtion Theory for Linear Operators," Springer verlag, New York Berlin, 1966.

4. J. KLAuder, Field structure through model studies, Acta Phys. Austriaca, to appear.

5. M. Reed And B. Simon, "Methods of Modern Mathematical Physics," Vol. I, Academic Press, New York, 1972.

6. B. Simon, Coupling constant analyticity for the anharmonic oscillator, Ann. Phys. 58 (1970), 76-136.

7. B. Simon, Essential self-adjointness of Schrödinger operators with singular potentials: A generalized Kalf-Walter-Schmincke theorem, Arch. Rat. Mech. Anal, to appear. 